\title{
Stimulated transitions in resonant atom Majorana mixing
}

\author{
José Bernabéu and Alejandro Segarra \\ Departament de Física Tè̀rica and IFIC, Universitat de València - CSIC, \\ Calle Dr. Moliner 50, E-46100 Burjassot, Spain \\ E-mail: Jose.Bernabeu@uv.es, Alejandro.Segarra@uv.es
}

ABSTRACT: Massive neutrinos demand to ask whether they are Dirac or Majorana particles. Majorana neutrinos are an irrefutable proof of physics beyond the Standard Model. Neutrinoless double electron capture is not a process but a virtual $\Delta L=2$ mixing between a parent ${ }^{A} Z$ atom and a daughter ${ }^{A}(Z-2)$ excited atom with two electron holes. As a mixing between two neutral atoms and the observable signal in terms of emitted two-hole X-rays, the strategy, experimental signature and background are different from neutrinoless double beta decay. The mixing is resonantly enhanced for almost degeneracy and, under these conditions, there is no irreducible background from the standard two-neutrino channel. We reconstruct the natural time history of a nominally stable parent atom since its production either by nature or in the laboratory. After the time periods of atom oscillations and the decay of the short-lived daughter atom, at observable times the relevant "stationary" states are the mixed metastable long-lived state and the non-orthogonal shortlived excited state, as well as the ground state of the daughter atom. We find that they have a natural population inversion which is most appropriate for exploiting the bosonic nature of the observed atomic transitions radiation. Among different observables of the atom Majorana mixing, we include the enhanced rate of stimulated X-ray emission from the long-lived metastable state by a high-intensity X-ray beam: a gain factor of 100 can be envisaged at current XFEL facilities. On the other hand, the historical population of the daughter atom ground state can be probed by exciting it with a current pulsed optical laser, showing the characteristic absorption lines: the whole population can be excited in a shorter time than typical pulse duration.

Keywords: Neutrino Physics, Beyond Standard Model, Global Symmetries

ArXiv ePrint: 1706.08328 


\section{Contents}

1 Introduction 1

2 The evolution Hamiltonian 5

3 Natural time history for initial $\left|{ }^{A} Z\right\rangle \quad 6$

4 Stimulated transitions $\quad 8$

4.1 Emission from $\left|\lambda_{L}\right\rangle \quad 8$

4.2 Absorption from $\left|A(Z-2)_{\text {g.s. }}\right\rangle \quad 10$

5 Conclusions 11

\section{Introduction}

The experimental evidence of neutrino oscillations is one of the most important discoveries in particle physics. Model-independent first evidences of neutrino oscillations were obtained in 1998 by the atmospheric neutrino experiment Super-Kamiokande [1], in 2002 by the solar neutrino experiment SNO [2], and later by accelerator and reactor neutrinos.

The existence of neutrino oscillations implies that neutrinos are massive particles and that the three flavor neutrinos $\nu_{e}, \nu_{\mu}, \nu_{\tau}$ are mixtures of the neutrinos with definite masses $\nu_{i}$ (with $\mathrm{i}=1,2,3$ ). The phenomenon of neutrino oscillations is being studied in a variety of experiments [3] which fully confirm this quantum phenomenon in different disappearance and appearance channels. The mixing matrix $U_{\mathrm{PMNS}}$ contains three mixing angles, already known, and one $\mathrm{CP}$ violating phase for flavor oscillations. Interacting neutrinos have lefthanded chirality.

Knowing that neutrinos are massive, the most fundamental open problem is the determination of the nature of neutrinos with definite mass: are they four-component Dirac particles with a conserved total lepton number $L$, distinguishing neutrinos from antineutrinos, or two-component truly neutral (no electric charge and no total lepton number) self-conjugate Majorana particles [4]? For Dirac neutrinos, like quarks and charged leptons, their masses can be generated in the Standard Model of particle physics by spontaneous breaking of the gauge symmetry with the Higgs scalar, if there were additional right-handed sterile neutrinos. But the Yukawa couplings would then be unnaturally small compared with all other fermions. A Majorana $\Delta L=2$ mass term, with the active lefthanded neutrinos only, leads to definite mass neutrinos with no definite charge. However, there is no way in the Standard Model able to generate this Majorana mass, so the important conclusion in fundamental physics arises: Majorana neutrinos are an irrefutable proof of physics beyond the Standard Model. Due to the Majorana condition of neutrinos 
with definite mass being their own antiparticles, Majorana neutrinos have additional CP violating phases [5-7] beyond the Dirac case.

Neutrino flavor oscillation experiments cannot answer the fundamental question of the nature of massive neutrinos, because in these flavor transitions the total lepton number $L$ is conserved. In order to probe whether neutrinos are Dirac or Majorana particles, we need to study observables violating the total lepton number $L$. The difficulty encountered in these studies is well illustrated by the so-called "confusion theorem" $[8,9]$, stating that in the limit of zero-mass there is no difference between Dirac and Majorana neutrinos. As all known neutrino sources produce highly relativistic neutrinos (except for the present cosmic neutrino background in the universe), the $\Delta L=2$ observables are highly suppressed.

Up to now, there is a consensus that the highest known sensitivity to small Majorana neutrino masses can be reached in experiments on the search of the $L$-violating neutrinoless double- $\beta$ decay process $(0 \nu \beta \beta)$

$$
{ }^{A} Z \rightarrow{ }^{A}(Z+2)+2 e^{-}
$$

where ${ }^{A} Z$ is a nucleus with atomic number $Z$ and mass number $A$. The two-neutrino double- $\beta$ decay process $(2 \nu \beta \beta)$ :

$$
{ }^{A} Z \rightarrow{ }^{A}(Z+2)+2 e^{-}+2 \bar{\nu}_{e}
$$

is allowed by the Standard Model for some even-even nuclei for which the single $\beta$ decay or electron capture is forbidden. The process (1.2) represents an irreducible background in the search of (1.1), which needs an excellent energy resolution in order to separate the definite peak in (1.1) from the high energy tail of the $2 e^{-}$spectrum in (1.2).

Dozens of experiments around the world are seeking out a positive signal of $0 \nu \beta \beta$. The most favorable decays for the experimental search are those with high mass difference between the ground state neutral atoms. The most sensitive limits at present are from GERDA-Phase II [10] for ${ }^{76} \mathrm{Ge}$, located at the Laboratori Nazionali del Gran Sasso (LNGS), and from KAMLAND-Zen [11] for ${ }^{136}$ Xe, located at the Kamioka Observatory. If the decay process is mediated by the exchange of light Majorana neutrinos, the mismatch between e-flavor neutrino and definite mass neutrinos $\nu_{i}$ in the Majorana propagator generates a decay amplitude proportional to the effective Majorana neutrino mass

$$
m_{\beta \beta} \equiv \sum_{i} U_{e i}^{2} m_{\nu_{i}}
$$

which is a coherent combination of the three neutrino masses. Its determination would then provide a measure of the absolute neutrino mass scale. The effective mass in eq. (1.3) depends on the mixing angles and two relative $\mathrm{CP}$ phases of the neutrinos in the $U_{\mathrm{PMNS}}$ mixing matrix. Assuming that neutrinos are Majorana particles, the present knowledge of mixing angles and neutrino mass differences, from neutrino flavor oscillations, produces the information $[12,13]$ condensed in figure 1 for the fundamental quantity $m_{\beta \beta}$.

Present experimental limits [14], indicated in figure 1, are approaching the interval of $m_{\beta \beta}$ values predicted for the inverse hierarchy in the neutrino mass spectrum, $\Delta m_{13}^{2}<0$. 


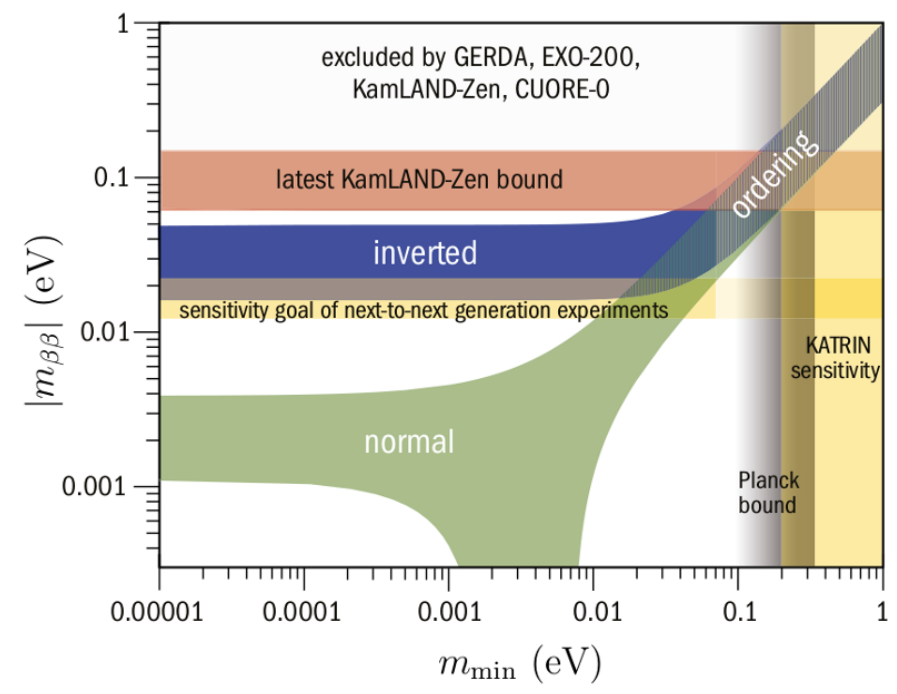

Figure 1. Allowed parameter space for a $0 \nu \beta \beta$ signal, as a function of the smallest neutrino mass, for both possible hierarchies. Current experimental bounds are also shown. Taken from [15].

The sign of $\Delta m_{13}^{2}$ is still an open question in neutrino physics and it is a subjet of current experimental interest $[16,17]$.

There is an alternative to $0 \nu \beta \beta$ by means of the mechanism of neutrinoless double electron capture $(0 \nu \mathrm{ECEC})$,

$$
{ }^{A} Z+2 e^{-} \rightarrow{ }^{A}(Z-2)^{*}
$$

This is actually a mixing between two states of two different neutral atoms differing in the total lepton number $L$ by two units, and the same baryonic number $A$, and not a process conserving energy and momentum in general. The daughter atom is in an excited state with two electron holes, and its decay provides the signal for (1.4). Ref. [18] first pointed out that the monumental coincidence of the initial energy of the parent atom and that of the intermediate excited atom would give rise to a large enhancement of the decay probability. The concept of resonant enhancement of $0 \nu \mathrm{ECEC}$ was further developed in $[19,20]$ for the exceptional circumstance of almost degeneracy between the parent and daughter atomic states in (1.4). The almost matching condition is fulfilled when the 2X-ray decay occurs through the tail of the width of the atomic state, as shown schematically in figure 2 . These works stimulated many experimental searches [21-31] of candidates when the remarkable trap technique for precision measurements of atomic masses became available.

The mixing amplitude was calculated in [20] from the diagram in figure 3 and, in a good approximation, it can be factorized leading to

$$
M_{21}=m_{\beta \beta}^{*}\left(\frac{G_{F} \cos \theta_{C}}{\sqrt{2}}\right)^{2}\left\langle F_{21}\right\rangle \frac{g_{A}^{2}}{2 \pi} M_{0 \nu}
$$

Here $G_{F}$ is the Fermi coupling constant, $\theta_{C}$ is the Cabibbo angle, $\left\langle F_{21}\right\rangle$ gives the probability amplitude of finding the two electrons in the nucleus, $M_{0 \nu}$ is the nuclear matrix element, 


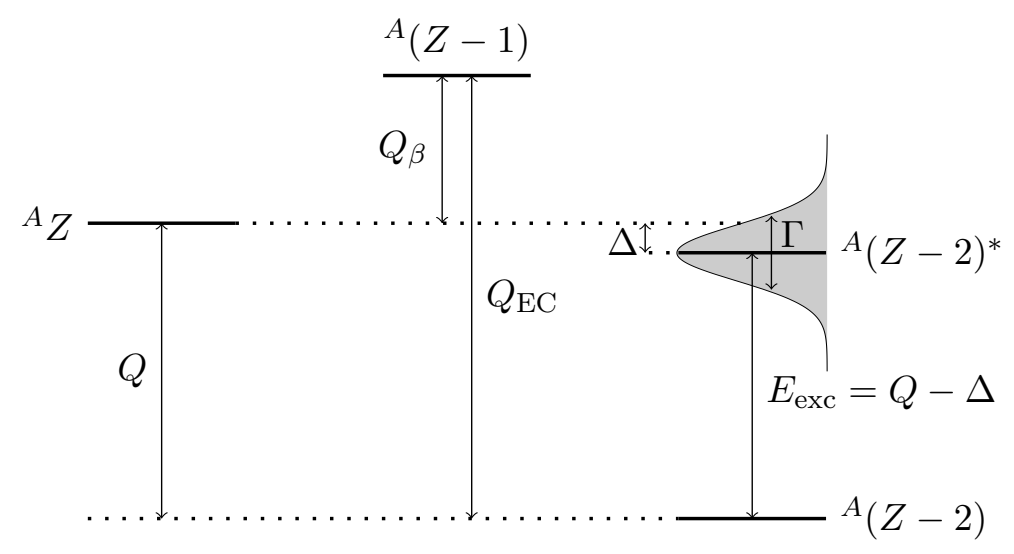

Figure 2. Schematic representation of the $\left\{{ }^{A} Z,{ }^{A}(Z-2)^{*}\right\}$ mixed atomic system.

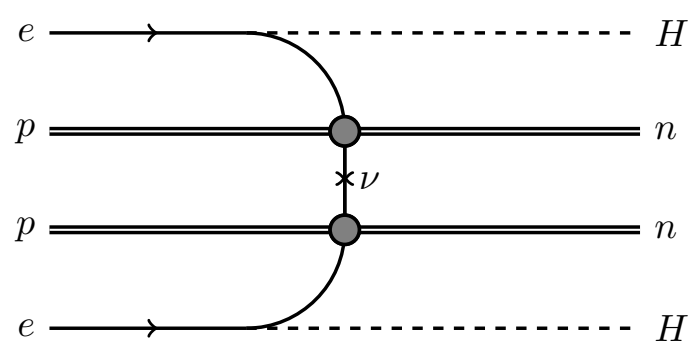

Figure 3. Feynman diagam for the Majorana mixing amplitude of ${ }^{A} Z$ atom in the ground state and the 2 -hole ${ }^{A}(Z-2)^{*}$ state.

which is of the order of the inverse nuclear radius, $g_{A}$ is the axial-vector nucleon coupling and the effective Majorana neutrino mass $m_{\beta \beta}$ appears as the complex conjugate of the expression (1.3) for neutrinoless double beta decay. The experimental activity in recent years has in turn stimulated the calculation [32-39] of the nuclear matrix elements for the cases of interest. A list of likely resonant transitions was provided in [35], excluding some of those previously suggested in [20]. After the improvements in measurements of atomic masses, the remaining candidates include ${ }^{152} \mathrm{Gd} \rightarrow{ }^{152} \mathrm{Sm},{ }^{164} \mathrm{Er} \rightarrow{ }^{164} \mathrm{Dy}$ and ${ }^{180} \mathrm{~W} \rightarrow{ }^{180} \mathrm{Hf}$ for atomic mixing to the daughter atom, with the nucleus in the ground state and having two holes in the inner atomic shells. More recent detailed analyses, using the state of the art in nuclear QRPA and IBM models, agree in the results, showing that the most promising known candidates are ${ }^{152} \mathrm{Gd}$ and ${ }^{180} \mathrm{~W}$.

The case of ${ }^{152} \mathrm{Gd} \rightarrow{ }^{152} \mathrm{Sm}$ mixing and decay is particularly attractive. The values of the relevant parameters are the experimental $\Delta=M_{1}-M_{2}=(0.91 \pm 0.18) \mathrm{keV}$ [21] for the masses of the parent " 1 " and daughter "2" atoms, $\Gamma=0.023 \mathrm{keV}$ [22] for the two-hole atomic width and the $Q$-value of the ground state to ground state transition $Q=(55.70 \pm 0.18) \mathrm{keV}[21]$. The theoretical mixing is [36, 39]

$$
\left|M_{21}\right|=10^{-24}\left[\frac{\left|m_{\beta \beta}\right|}{0.1 \mathrm{eV}}\right] \mathrm{eV} \text {. }
$$


As seen, the optimal resonant enhancement condition is still off by at least a factor 30 , implying a loss of 3 orders of magnitude in the expected X-ray rate from the parent atom.

In section 2 we set the formalism for the two-state atom mixing [33, 35] using a nonnormal non-Hermitian Hamiltonian and obtain the non-orthogonal states with definite time evolution: one metastable mixed state and one short-lived mixed state. In section 3 we develop the natural time history of an initial parent ground state atom, a history which is governed by different time scales of oscillation, lifetime of the short-lived state and lifetime of the metastable state, thus obtaining the expected natural populations, at present observable times, of the three states involved including the ground state of the daughter atom. Stimulated by the natural population inversion, we contemplate in section 4 the prospects that could be open by both an enhanced rate of stimulated X-ray emission from the metastable state and the absorption from the ground state daughter atom. In section 5 we present our conclusions and outlook.

\section{The evolution Hamiltonian}

In the basis of the $\left|{ }^{A} Z\right\rangle$ and $\left|{ }^{A}(Z-2)^{*}\right\rangle$ states, which we'll refer to as 1 and 2 , the dynamics of this two-state system of interest is governed by the Hamiltonian

$$
\mathbb{H}=\mathbb{M}-\frac{i}{2} \mathbb{\Gamma}=\left[\begin{array}{cc}
M_{1} & M_{21}^{*} \\
M_{21} & M_{2}
\end{array}\right]-\frac{i}{2}\left[\begin{array}{cc}
0 & 0 \\
0 & \Gamma
\end{array}\right],
$$

with a Majorana $\Delta L=2$ mass mixing $M_{21}$ as given by eq. (1.5). The anti-Hermitian part of this Hamiltonian is due to the instability of $\left|{ }^{A}(Z-2)^{*}\right\rangle$, which de-excites into $\left|{ }^{A}(Z-2)_{\text {g.s. }}\right\rangle$, external to the two-body system in eq. (2.1), emitting its two-hole characteristic X-ray spectrum. The appropriate non-Hermitian Hamiltonian formalism for describing the mixing of a two-state unstable system is known since Weisskopf-Wigner [40] and it was used in many instances. It has been employed [33, 35] with the objective of reproducing the rate, induced by atom mixing, as previously given in ref. [20].

Besides being non-Hermitian, $\mathbb{H}$ is not a normal operator, i.e. $[\mathbb{M}, \mathbb{\Gamma}] \neq 0$. As a consequence, $\mathbb{M}$ and $\mathbb{\Gamma}$ are not compatible. The states of definite time evolution, eigenstates of $\mathbb{H}$, have complex eigenvalues and are given in non-degenereate perturbation theory [41] by

$$
\begin{array}{ll}
\left|\lambda_{L}\right\rangle=|1\rangle+\alpha|2\rangle, & \lambda_{L} \equiv E_{L}-\frac{i}{2} \Gamma_{L}=M_{1}+|\alpha|^{2}\left[\Delta-\frac{i}{2} \Gamma\right], \\
\left|\lambda_{S}\right\rangle=|2\rangle-\beta^{*}|1\rangle, & \lambda_{S} \equiv E_{S}-\frac{i}{2} \Gamma_{S}=M_{2}-\frac{i}{2} \Gamma-|\alpha|^{2}\left[\Delta-\frac{i}{2} \Gamma\right],
\end{array}
$$

with $\Delta=M_{1}-M_{2}$. As seen in eq. (2.2), $\Gamma_{L, S}$ are not the eigenvalues of the $\mathbb{\Gamma}$ matrix. The eigenstates are modified at first order in $M_{21}$,

$$
\alpha=\frac{M_{21}}{\Delta+\frac{i}{2} \Gamma}, \quad \quad \beta=\frac{M_{21}}{\Delta-\frac{i}{2} \Gamma},
$$

so the "stationary" states of the system don't have well-defined atomic properties: both the number of electrons and their atomic properties are a superposition of $Z$ and $Z-2$. 
Also, these states are not orthogonal - their overlap is given by

$$
\left\langle\lambda_{S} \mid \lambda_{L}\right\rangle=\alpha-\beta=-i \frac{M_{21} \Gamma}{\Delta^{2}+\frac{1}{4} \Gamma^{2}},
$$

with its non-vanishing value due to the joint presence of the mass mixing $M_{21}$ and the decay width $\Gamma$. Notice that $\operatorname{Im}\left(M_{21}\right)$ originates a real overlap.

As seen in eq. (2.2), the modifications in the corresponding eigenvalues appear at second order in $\left|M_{21}\right|$ and they are equidistant with opposite sign. Since these corrections are small, from now on we will use the values

$$
\begin{aligned}
& E_{L} \approx M_{1}, \quad E_{S} \approx M_{2}, \\
& \Gamma_{L} \approx|\alpha|^{2} \Gamma, \quad \Gamma_{S} \approx \Gamma .
\end{aligned}
$$

The only relevant correction at order $|\alpha|^{2}$ is the one to $\Gamma_{L}$, since $|1\rangle$ was a stable state even if it's small, the mixing produces a non-zero decay width.

This result shows that, at leading order, the Majorana mixing becomes observable through $\Gamma_{L} \propto|\alpha|^{2}$. The value of $\alpha$ in eq. (2.3) emphasizes the relevance of the condition $\Delta \sim \Gamma$, which produces a resonant enhancement [20] of the effect of the $\Delta L=2$ mass mixing $M_{21}$.

\section{$3 \quad$ Natural time history for initial $\left|{ }^{A} Z\right\rangle$}

As seen in eq. (2.2), the states $\left|{ }^{A} Z\right\rangle$ and $\left|{ }^{A}(Z-2)^{*}\right\rangle$ are not the stationary states of the system. For an initially prepared $\left|{ }^{A} Z\right\rangle$, the time history is far from trivial and the appropriate language to describe the system short times after is that of atom oscillations [33] between $\left|{ }^{A} Z\right\rangle$ and $\left|{ }^{A}(Z-2)^{*}\right\rangle$ due to the interference of the amplitudes through $\left|\lambda_{S}\right\rangle$ and $\left|\lambda_{L}\right\rangle$ in the time evolution. The time-evolved $\left|{ }^{A} Z\right\rangle$ state becomes

$$
\left|{ }^{A} Z(t)\right\rangle=e^{-i \lambda_{L} t}\left|\lambda_{L}\right\rangle-\alpha e^{-i \lambda_{S} t}\left|\lambda_{S}\right\rangle
$$

and the appearance probability is then given by

$$
\left|\left\langle{ }^{A}(Z-2)^{*} \mid{ }^{A} Z(t)\right\rangle\right|^{2}=|\alpha|^{2}\left\{1+e^{-\Gamma t}-2 e^{-\frac{1}{2} \Gamma t} \cos (\Delta \cdot t)\right\},
$$

with an oscillation angular frequency $|\Delta|$. The characteristic oscillation time $\tau_{\text {osc }}=$ $2 \pi|\Delta|^{-1}$ is the shortest time scale in this system. For $t \ll \tau_{\text {osc }}$, one has

$$
\left|\left\langle{ }^{A}(Z-2)^{*} \mid{ }^{A} Z(t)\right\rangle\right|^{2} \approx\left|M_{21}\right|^{2} t^{2}
$$

induced by the mass mixing.

The next shortest characteristic time in this system is the decay time $\tau_{S}=\Gamma^{-1}$, associated to the $\left|\lambda_{S}\right\rangle$ state. For $\tau_{\text {osc }} \ll t \ll \tau_{S}$, the only change with respect to eq. (3.2) is that the interference region disappears, and the two slits $\left|\lambda_{L}\right\rangle$ and $\left|\lambda_{S}\right\rangle$ in (3.1) contribute incoherently,

$$
\left.\left|{ }^{A}(Z-2)^{*}\right|^{A} Z(t)\right\rangle\left.\right|^{2} \approx|\alpha|^{2}(2-\Gamma t) .
$$




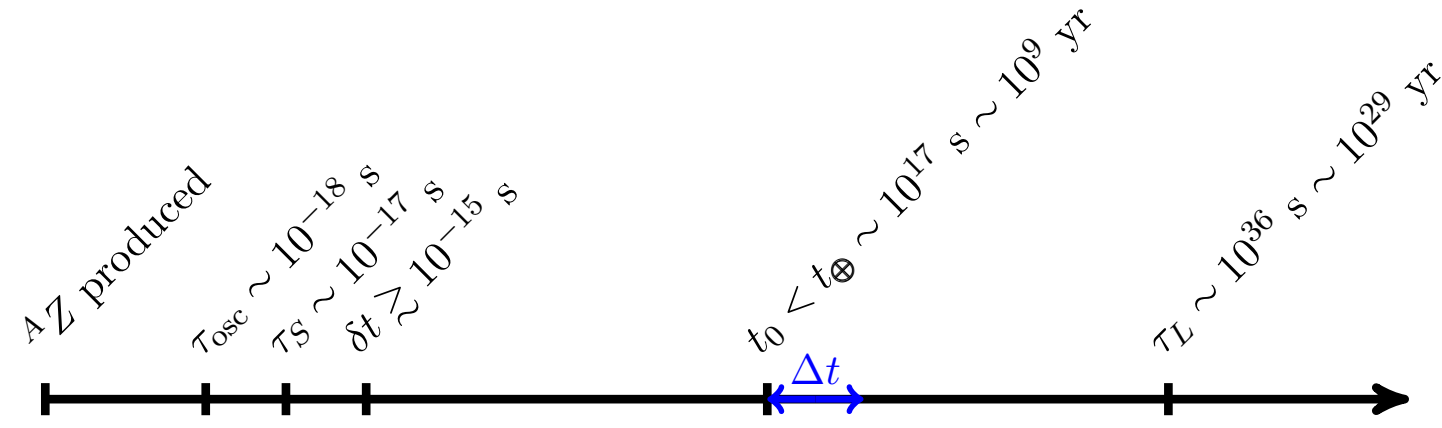

Figure 4. Timeline of the mixed atomic system. The $\tau_{\text {osc }}, \tau_{S}$ and $\tau_{L}$ values are for ${ }^{152} \mathrm{Gd} \rightarrow{ }^{152} \mathrm{Sm}$, whereas $\delta t$ is a typical time resolution, $t_{0}$ is the age of the sample ore, $t_{\oplus}$ is the age of the Earth and $\Delta t$ is the observation time in an actual experiment.

For $t \gg \tau_{S}$, the contribution of $\left|\lambda_{S}\right\rangle$ disappears and the appearance probability simply becomes

$$
\left|\left\langle\left.{ }^{A}(Z-2)^{*}\right|^{A} Z(t)\right\rangle\right|^{2}=|\alpha|^{2}
$$

In other words, the initially prepared $\left|{ }^{A} Z\right\rangle$ state evolves towards the stationary metastable state $\left|\lambda_{L}\right\rangle$,

$$
\left|{ }^{A} Z(t)\right\rangle \rightarrow e^{-i \lambda_{L} t}\left|\lambda_{L}\right\rangle
$$

with the long lifetime $\tau_{L}=\Gamma_{L}^{-1}$ from eq. (2.5). For a realistic time resolution $\delta t$ in an actual experiment, this regime is the interesting one, with the behavior in eq. (3.6). As shown in figure 4 , the different time scales involved in this problem are thus

$$
\tau_{\text {osc }} \ll \tau_{S} \ll \delta t \ll t \ll \tau_{L}
$$

where $t$ refers to the elapsed time since the production of ${ }^{A} Z$, either by nature or in the lab - given the smallness of the mixing, the metastability of the state (3.6) is valid even for cosmological times. Therefore, for any time between the two scales $\tau_{S}$ and $\tau_{L}$, the populations of the three states involved are given by the probabilities

$$
\tau_{S} \ll t \ll \tau_{L} \Longrightarrow\left\{\begin{aligned}
P_{L}(t) & \approx 1-\Gamma_{L} t \\
P_{S}(t) & \approx 0 \\
P_{\text {g.s. }}(t) & \approx|\alpha|^{2} \Gamma t
\end{aligned}\right.
$$

where $P_{\text {g.s. }}(t)$ refers to the population of the ground state of the ${ }^{A}(Z-2)$ atom after the decay of the unstable "stationary" state $\left|\lambda_{S}\right\rangle$ in (3.1), with rate $\Gamma$. No matter whether $t$ refers to laboratory or cosmological times, the linear approximation in $t$ is excellent.

With this spontaneous evolution of the system, an experiment beginning its measurements a time $t_{0}$ after the ${ }^{A} Z$ was produced will probe the three-level system with relative populations $P_{L} \approx 100 \%, P_{S} \approx 0, P_{\text {g.s. }} \approx|\alpha|^{2} \Gamma t_{0}$. We discover two methods, involving 
the third state beyond the mixed states, ${ }^{1}$ to be sensitive to the resonant Majorana mixing of atoms:

- Spontaneous emission from the metastable state to the daughter atom ground state. The population in the upper level $\left|\lambda_{L}\right\rangle$, as shown in eq. (3.8a), decreases with time as $P_{L}(\Delta t) \approx 1-\Gamma_{L} \Delta t$, where $\Delta t=t-t_{0}$, due to the decay of the metastable "stationary" state $\left|\lambda_{L}\right\rangle$ to $\left|{ }^{A}(Z-2)_{\text {g.s. }}\right\rangle$. This process is associated to the spontaneous emission of X-rays with a rate $\Gamma_{L}$, considered in the literature after the concept of resonant mixing was introduced in ref. [20]. For one mole of ${ }^{152} \mathrm{Gd}$, the X-ray emission rate would be of order $10^{-12} \mathrm{~s}^{-1} \sim 10^{-5} \mathrm{yr}^{-1}$. The initial state in the transition at observable times, being $\left|\lambda_{L}\right\rangle$, tells us that the total energy of the two-hole X-ray radiation is displaced by $\Delta$ with respect to the characteristic $\left|{ }^{A}(Z-2)^{*}\right\rangle \rightarrow\left|{ }^{A}(Z-2)_{\text {g.s. }}\right\rangle$ $\mathrm{X}$-ray spectrum, i.e. its energy release is the $Q$-value between the two atoms in their ground states (as seen in figure 2).

- Daughter atom population. The presence of the daughter atom in the parent ores (see eq. (3.8c)), can be probed e.g. by geochemical methods. For one mole of the nominally stable ${ }^{152} \mathrm{Gd}$ isotope produced at the time of the Earth formation, the values in figure 4 would predict an accumulated number of order $10^{4}{ }^{152} \mathrm{Sm}$ atoms. This observable could be of interest for cosmological times $t_{0}$ since, contrary to $\beta \beta$ decay, in the ECEC case there is no irreducible background from a $2 \nu$ channel for a resonant atom mixing.

We would like to emphasize that, even though $\Gamma_{L}=|\alpha|^{2} \Gamma$ ensures the probability conservation, an interpretation of eqs. (3.8a) and (3.8c) in different terms is of interest. On the one hand, $\Gamma_{L}$ is the rate for the decay of $\left|\lambda_{L}\right\rangle$ at any time $t$, which accounts for the observable 1 . On the other hand, the population of the daughter atom in the ground state is obtained from the mixing probability leading to $\left|\lambda_{S}\right\rangle$ in eq. (3.1) at all times, given by $|\alpha|^{2}$, times its decay rate to the ground state, $\Gamma_{S}=\Gamma$. This mixing $\times$ Decay temporal evolution explains the non-zero population of ${ }^{A}(Z-2)_{\text {g.s. }}$, producing the second observable.

\section{Stimulated transitions}

\subsection{Emission from $\left|\lambda_{L}\right\rangle$}

A careful reading of eqs. (3.8) shows that the metastable state $\left|\lambda_{L}\right\rangle$ and the ground state $\left|{ }^{A}(Z-2)_{\text {g.s. }}\right\rangle$ have a natural population inversion, with an overwhelming abundance of the

\footnotetext{
${ }^{1}$ One may wonder whether there is, for $\Delta>0$, a spontaneous emission of lower energy X-rays from $\left|\lambda_{L}\right\rangle$ to $\left|\lambda_{S}\right\rangle$ leading to a regeneration of the short-lived mixed state. Observing in (2.2) the atom mixing of these states, the dynamics of this process would be that of the Compton amplitudes for the $Z$ and $(Z-2)^{*}$ atoms, whereas the kinematics corresponds to two-photon emission instead of scattering. At these intermediate energies between atomic and nuclear physics, the Compton amplitude $T_{2 \gamma}$ can be taken to be an incoherent sum of the electron contributions [42, 43],$$
T_{2 \gamma}=\alpha T_{2 \gamma}^{Z-2}-\beta T_{2 \gamma}^{Z} \quad ; \quad T_{2 \gamma}^{Z}=\frac{Z e^{2}}{m}\left(\boldsymbol{\epsilon}^{\prime *} \cdot \boldsymbol{\epsilon}^{*}\right)
$$

where $m$ is the electron mass and $\boldsymbol{\epsilon}$ the polarization vectors of the photons. A straightforward calculation of the rate for this e.m. $\left|\lambda_{L}\right\rangle \rightarrow\left|\lambda_{S}\right\rangle$ transition, when compared to the transition to the daughter atom ground state, gives a branching ratio of the order $10^{-7}$.
} 
long-lifetime upper level of the system. This result suggests the exploitation of the bosonic properties of the X-radiation, used as a signal of the Majorana mixing in this problem, and considering the external action of an X-ray beam to stimulate the emission from the metastable level to the ground state of this atomic system.

Stimulated radiation for the emission from $\left|\lambda_{L}\right\rangle$ to the ground state $\left.\left.\right|^{A}(Z-2)_{\text {g.s. }}\right\rangle$ could then enhance the rate and we present an estimate of the gain which could be envisaged in future facilities of X-ray beams. A setup with an incident pulsed beam allows the observation of low rate events in directions outside the beam direction and the control of background conditions in the absence of the beam. Therefore, one discovers a third observable

- Stimulated emission from $\left|\lambda_{L}\right\rangle$ to $\left|{ }^{A}(Z-2)_{\text {g.s. }}\right\rangle$. The natural population inversion between the ground state and the metastable "stationary" state $\left|\lambda_{L}\right\rangle$ gives raise to the possibility of stimulating the decay $\left|\lambda_{L}\right\rangle \rightarrow\left|{ }^{A}(Z-2)_{\text {g.s. }}\right\rangle$. The experimental signature of this process would be the emission of X-rays with total energy equal to the $Q$-value of the process, just like in the first observable of spontaneous emission.

For the emission between the two levels $\left|\lambda_{L}\right\rangle \rightarrow\left|{ }^{A}(Z-2)_{\text {g.s. }}\right\rangle$ of radiation with angular frequency $\omega$, stimulated radiation is described in terms of the Einstein coefficients [44] with an induced rate $^{2}$

$$
\frac{\mathrm{d} N_{L}}{\mathrm{~d} t}=-\frac{\pi^{2} c^{3}}{\hbar \omega^{3}} \rho_{\omega} \Gamma_{L} N_{L},
$$

where $N_{L}$ is the population of the upper metastable $\left|\lambda_{L}\right\rangle$ level, $\Gamma_{L}$ its width and $\rho_{\omega}$ is the energy density of the beam per unit of angular frequency, i.e.

$$
\rho_{\omega}=\frac{\mathrm{d} E}{c \mathrm{~d} t \mathrm{~d} S \mathrm{~d} \omega} .
$$

Therefore, this observable is enhanced with respect to the first one by a gain factor

$$
G=\frac{\pi^{2} c^{3}}{\hbar \omega^{3}} \rho_{\omega},
$$

which is the ratio between the stimulated and spontaneous emission rates.

In order to produce a sizable gain, one should devise a setup with as large a $\rho_{\omega}$ as possible. The transition energy of this system is of order tens of keV, so a high-luminosity X-ray beam is mandatory. Such high-energy beams are produced at free-electron laser (FEL) facilities, through a kind of laser consisting of very-high-speed electrons moving freely through a magnetic structure. Free-electron lasers are tunable and have the widest frequency range of any laser type, currently ranging in wavelength from microwaves, through terahertz radiation and infrared, to the visible spectrum, ultraviolet, and X-ray. The highest frequencies are obtained in XFEL facilities like the running SLAC Linac Coherent Light Source (LCLS) and the commissioned European XFEL (EXFEL) at DESY.

The determination of the gain factor one could achieve in these facilities is clearer after rewriting the spectral energy density (4.2) in terms of beam parameters,

$$
\rho_{\omega}=\frac{\hbar}{c} \frac{\mathrm{d} N}{\mathrm{~d} t \mathrm{~d} S}\left[\frac{\mathrm{d} \omega}{\omega}\right]^{-1}
$$

\footnotetext{
${ }^{2}$ For the sake of clarity, throughout this discussion we keep all $\hbar$ and $c$ factors.
} 
Taking $\mathrm{d} N / \mathrm{d} t$ as the number of photons per pulse duration, $\mathrm{d} S$ as the beam section and $\mathrm{d} \omega / \omega$ as the full width half maximum (FWHM) spectrum width, one finds the gain factor

$$
G=\hbar(\hbar c)^{2} \frac{\pi^{2}}{(\hbar \omega)^{3}} \frac{\mathrm{d} N}{\mathrm{~d} t \mathrm{~d} S}\left[\frac{\mathrm{d} \omega}{\omega}\right]^{-1}
$$

to be written in terms of clearly defined beam properties, where $\mathrm{d} N / \mathrm{d} t \mathrm{~d} S$ is the luminosity $\mathcal{L}$ of the beam.

At EXFEL, a sound simulation of the conditions of the machine [45] gives, for typical energies of tens of $\mathrm{keV}$, the expected number of photons per pulse duration $d N / d t=$ $10^{10} \mathrm{fs}^{-1}$ and the spectral width $\mathrm{d} \omega / \omega=1.12 \times 10^{-3}$. Nanofocusing of this X-ray FELs has been contemplated [46]; using a beam spot of the order of $100 \mathrm{~nm}$ would lead to a gain factor from (4.5) of $G \sim 100$. The continuous interaction of these X-rays with a mole of ${ }^{152} \mathrm{Gd}$ atoms would provide a stimulated rate of order $10^{-10} \mathrm{~s}^{-1} \sim 10^{-3} \mathrm{yr}^{-1}$.

One should notice that this rate of events assumes constant irradiation of the whole target. The straightforward setup of a cylindrical target alongside the pulsating X-ray beam presents different issues. Most notoriously, pulsed beams limit the enhancing time to a fraction of the running time. EXFEL manages to produce $2.7 \times 10^{4}$ pulses per second, so that the fraction of effective time is of order $10^{-9}$; LCLS-II expects to produce pulses at $1 \mathrm{MHz}$, increasing this number by two orders of magnitude, but still far away from a promising factor. Furthermore, radiation of these energies has an attenuation length in Gd of tens of microns, limiting the amount of material one could use to a fraction of a mole. This setup has the general drawback that the high energy density effect associated with the small beam spot size is lost when considering the small interaction volume.

The attenuation of the beam is associated to its interaction with the sample, which is dominated by the photoelectric effect and, to a lesser extent, inelastic Compton scattering, leading to ionization. Successive interactions of the secondary electrons will heat the material. A recent simulation [47] of this effect under realistic experimental conditions on a cylindrical target, assuming the extreme limit that the whole absorption power is converted into heating power, shows that a temperature of about $700^{\circ} \mathrm{C}$ is reached for an incoming beam of spot size $100 \mathrm{~nm}$ and an average of $10^{14} \mathrm{X}$-ray photons/s. Since this temperature is proportional to the flux, in all high-flux experiments like the one contemplated in this work, the small-interaction-volume target is actually destroyed. The design of a macroscopic sample whith a very large number of thermally isolated micro-targets, built on a plane in transversal motion synchronized with the pulse frequency of the beam, is a subject of current interest [48]. The use of this approach in order to stimulate the $\Delta L=2$ emission rate should be explored after a suitable candidate is found. On the other hand, the limiting factors in the expected integrated rate of events also suggest an alternative ingenuity program more in the line of micro-particles inserted into a dreamed $\mathrm{X}$-ray resonant cavity.

\subsection{Absorption from $\left|A(Z-2)_{\text {g.s. }}\right\rangle$}

A different observable may also be considered. The existing population of the daughter atom in its ground state is, by itself, a signal of the atom Majorana mixing, as discussed 
in the previous section as a relic of the previous history with an initial parent atom. In addition, this population can be identified by using an intense photon beam, leading to the characteristic absorption spectrum of the daughter atom and its subsequent decay to the ground state.

- Stimulated absorption spectrum of the daughter atom. In the presence of a light beam, the daughter atom population would absorb those characteristic frequencies corresponding to its energy levels, which would then de-excite emitting light of the same frequency. In the case of the one mole ${ }^{152} \mathrm{Gd}$ ore that we mentioned in the previous section, all $10^{4} \mathrm{Sm}$ atoms could be easily excited to any of its $\sim 1 \mathrm{eV}$ levels using a standard pulsed laser of order 100 fs pulse duration, with a mean power of $5 \mathrm{~W}$ and a pulse rate of $100 \mathrm{MHz}$.

Notice that these numbers imply, for a laser with FWHM spot size $\sim 40 \mu \mathrm{m}$, an absorption rate

$$
\left.\frac{\mathrm{d} N_{\text {g.s. }}}{\mathrm{d} t}\right|_{\mathrm{abs}}=-60 \% N_{\text {g.s. }}\left[\frac{100 \mathrm{~ns}}{\tau}\right] \mathrm{fs}^{-1} .
$$

Since Sm levels have lifetimes between $10-1000$ ns [49], one expects to excite them all during the 100 fs pulse. Disentangling the parent and daughter lines should not be difficult - the relatively small number of atomic absorption lines (compared to atomic emission lines) and their narrow width (a few pm) make spectral overlap rare, not being expected between $Z$ and $(Z-2)$ atoms.

It is worth noting from the results of this section that the bosonic nature of the atomic radiation is a property that can help in getting observable rates of the atom Majorana mixing, including the stimulated X-ray emission from the parent atom as well as the detection of the presence of the daughter atoms by means of its characteristic absorption lines. The actual values correspond to the specific case of ${ }^{152} \mathrm{Gd} \rightarrow{ }^{152} \mathrm{Sm}$, which is still off the resonance condition by at least a factor 30, implying a factor $10^{3}$ in the rates.

\section{Conclusions}

Neutrinoless double electron capture in atoms is a quantum mixing mechanism between the neutral atoms ${ }^{A} Z$ and ${ }^{A}(Z-2)^{*}$ with two electron holes. It becomes allowed for Majorana neutrino mediation responsible of this $\Delta L=2$ transition. This Majorana mixing leads to the X-ray de-excitation of the $\left|{ }^{A}(Z-2)^{*}\right\rangle$ daughter atomic state which, under the resonance condition, has no Standard Model background from the two-neutrino decay.

The intense experimental activity looking for atomic candidates satisfying the resonance condition by means of precise measurements of atomic masses, thanks to the trapping technique, has already led to a few cases of remarkable enhancement effects and there is still room for additional adjustements of the resonance condition. With this situation, it is important to understand the complete time evolution of an atomic state since its inception and whether one can find, from this information, different signals of the Majorana mixing, including the possible enhancement due to the bosonic nature of atomic transition radiation. These points have been addressed in this work. 
The effective Hamiltonian for the two mixed atomic states leads to definite nonorthogonal states of mass and lifetime, each of them violating global lepton number, one being metastable with long lifetime and the other having a short lifetime. For an initial atomic state there are time periods of atom oscillations, with frequency the mass difference, and the decay of the short lived state, which are not observable for present time resolutions. For observable times, the system of the two atoms has three relevant states for discussing transitions: one highly populated state with long lifetime, one empty state with short lifetime and the ground state of the daughter atom with a small population as a result of the past history. As a consequence, this is a case of natural population inversion suggesting the possibility of stimulated radiation transitions besides the natural spontaneous X-ray emission.

The gain factor for stimulated emission due to a present radiation energy density per unit frequency has been adapted to the case of an X-ray beam in terms of conventional parameters like its luminosity, the energy and the spectrum width. Using simulated previsions of the now commissioned European XFEL facility, with a beam spot size of $100 \mathrm{~nm}$, we obtain an expected gain of 100 in the X-ray emission rate from the metastable long-lived state. This substantial gain by stimulating the X-ray emission from $\left|\lambda_{L}\right\rangle$ to $\left|{ }^{A}(Z-2)_{\text {g.s. }}\right\rangle$ for interacting $\mathrm{X}$-ray photons with atoms is, however, not exploited in a straightforward setup of a single pulsed beam directed towards a cylindrical target. The limiting factors of the pulse frequency and the small interaction volume are suppressing that ideal benefit for the integrated number of events.

The small population of the ground state daughter atom at observable times would be by itself a proof of the atomic Majorana mixing, given the absence of the standard two neutrino decay of the parent atom. Besides other geochemical methods, absorption rates and the subsequent emission by an intense photon beam for the daughter atom, due to its non-vanishing population of the ground state, can be clearly contemplated.

The results obtained in this work demonstrate that the knowledge of the time history of a nominally stable atom since its inception can be a source of inspiration to find appropriate observables in the search of evidence for $\Delta L=2$ double electron capture. The natural population inversion at observable times suggests stimulating the X-ray emission with gain factors which could become significant for appropriate setups. On the other hand, the presence of the daughter atom ground state can be signaled by looking for its characteristic absorption spectrum. Taking into account the ongoing searches for new isotope candidates with a better fulfillment of the resonance condition, it remains to be seen whether these processes, with the ideas on stimulating the transitions, could become actual alternatives in the quest for the Dirac/Majorana nature of neutrinos.

\section{Acknowledgments}

The authors would like to acknowledge scientific discussions with, and advice from, Massimo Altarelli, Michael Block, Albert Ferrando, Gaston Garcia and Pablo Villanueva. This research has been supported by MINECO Project FPA 2014-54459-P, Generalitat Valenciana Project GV PROMETEO II 2013-017 and Severo Ochoa Excellence Centre Project SEV 2014-0398. A.S. acknowledges the MECD support through the FPU14/04678 grant. 
Open Access. This article is distributed under the terms of the Creative Commons Attribution License (CC-BY 4.0), which permits any use, distribution and reproduction in any medium, provided the original author(s) and source are credited.

\section{References}

[1] Super-Kamiokande collaboration, Y. Fukuda et al., Evidence for oscillation of atmospheric neutrinos, Phys. Rev. Lett. 81 (1998) 1562 [hep-ex/9807003] [INSPIRE].

[2] SNO collaboration, Q.R. Ahmad et al., Direct evidence for neutrino flavor transformation from neutral current interactions in the Sudbury Neutrino Observatory, Phys. Rev. Lett. 89 (2002) 011301 [nucl-ex/0204008] [INSPIRE].

[3] M. Mezzetto Experimental outlook, in Neutrino 2016, July 4-9, London, U.K. (2016).

[4] E. Majorana, Teoria simmetrica dell'elettrone e del positrone, Nuovo Cim. 14 (1937) 171 [INSPIRE].

[5] S.M. Bilenky, J. Hosek and S.T. Petcov, On oscillations of neutrinos with Dirac and Majorana Masses, Phys. Lett. 94B (1980) 495 [InSPIRE].

[6] M. Doi, T. Kotani, H. Nishiura, K. Okuda and E. Takasugi, CP violation in Majorana neutrinos, Phys. Lett. 102B (1981) 323 [INSPIRE].

[7] J. Bernabéu and P. Pascual, CP properties of the leptonic sector for Majorana neutrinos, Nucl. Phys. B 228 (1983) 21 [inSPIRE].

[8] C. Ryan and S. Okubo, On the equivalence of the Majorana and two-component theories of the neutrino, Nuovo Cim. Suppl. 2 (1964) 234.

[9] K.M. Case, Reformulation of the Majorana theory of the neutrino, Phys. Rev. 107 (1957) 307 [INSPIRE].

[10] GERDA collaboration, V. D'Andrea, Status Report of the GERDA Phase II Startup, arXiv: 1604.05016 [inSPIRE].

[11] KamLAnD-Zen collaboration, A. Gando et al., Search for Majorana Neutrinos near the Inverted Mass Hierarchy Region with KamLAND-Zen, Phys. Rev. Lett. 117 (2016) 082503 [arXiv: 1605.02889] [INSPIRE].

[12] F. Vissani, Signal of neutrinoless double beta decay, neutrino spectrum and oscillation scenarios, JHEP 06 (1999) 022 [hep-ph/9906525] [INSPIRE].

[13] F. Feruglio, A. Strumia and F. Vissani, Neutrino oscillations and signals in beta and Onu2beta experiments, Nucl. Phys. B 637 (2002) 345 [hep-ph/0201291] [INSPIRE].

[14] F. Deppisch, Looking for lepton number violation, in Neutrino 2016, July 4-9, London, U.K. (2016).

[15] S. Pascoli, A portal to new physics, CERN courier, July-August (2016).

[16] S. Wren, Neutrino mass ordering studies with PINGU and IceCube/DeepCore, arXiv:1604.08807 [INSPIRE].

[17] A. Kouchner, KM3NeT - ORCA: measuring the neutrino mass ordering in the Mediterranean, J. Phys. Conf. Ser. 718 (2016) 060230.

[18] R.G. Winter, Double K capture and single K capture with positron emission, Phys. Rev. 100 (1955) 142 [INSPIRE]. 
[19] R.A. Eramzhian, G. Mitselmakher and M.B. Voloshin, Conversion of an atomic electron into a positron and double beta+ decay, JETP Lett. 35 (1982) 656 [INSPIRE].

[20] J. Bernabeu, A. De Rujula and C. Jarlskog, Neutrinoless double electron capture as a tool to measure the $\nu_{e}$ mass, Nucl. Phys. B 223 (1983) 15 [INSPIRE].

[21] S. Eliseev et al., Resonant enhancement of neutrinoless double-electron capture in Gd-152, Phys. Rev. Lett. 106 (2011) 052504 [InSPIRE].

[22] J.L. Campbell and T. Papp, Widths of the atomic K-N7 levels, Atom. Data Nucl. Data Tables 77 (2001) 1.

[23] V.S. Kolhinen et al., Accurate $Q$ value for the ${ }^{74}$ Se double-electron-capture decay, Phys. Lett. B 684 (2010) 17 [INSPIRE].

[24] A.S. Barabash, P. Hubert, A. Nachab and V. Umatov, Search for $\beta^{+} E C$ and ECEC processes in ${ }^{74} \mathrm{Se}$, Nucl. Phys. A 785 (2007) 371 [hep-ex/0610046] [INSPIRE].

[25] A.S. Barabash et al., Search for $\beta^{+} E C$ and ECEC processes in ${ }^{112} S n$ and $\beta^{-} \beta^{-}$decay of ${ }^{124}$ Sn to the excited states of ${ }^{124}$ Te, Nucl. Phys. A 807 (2008) 269 [arXiv:0804.3849] [INSPIRE].

[26] S. Eliseev et al., Q values for neutrinoless double-electron capture in ${ }^{96} R u,{ }^{162}$ Er and ${ }^{168} Y b$, Phys. Rev. C 83 (2011) 038501 [INSPIRE].

[27] V.S. Kolhinen et al., On the resonant neutrinoless double-electron-capture decay of ${ }^{136} \mathrm{Ce}$, Phys. Lett. B 697 (2011) 116 [INSPIRE].

[28] M. Goncharov et al., Probing the nuclides ${ }^{102} \mathrm{Pd},{ }^{106} \mathrm{Cd}$ and ${ }^{144} \mathrm{Sm}$ for resonant neutrinoless double-electron capture, Phys. Rev. C 84 (2011) 028501 [INSPIRE].

[29] C. Droese et al., Probing the nuclide ${ }^{180} \mathrm{~W}$ for neutrinoless double-electron capture exploration, Nucl. Phys. A 875 (2012) 1 [arXiv:1111.6377] [INSPIRE].

[30] M.F. Kidd, J.H. Esterline and W. Tornow, Double-electron capture on ${ }^{112}$ Sn to the excited $1871 \mathrm{keV}$ state in ${ }^{112} \mathrm{Cd}$ : a possible alternative to double-beta decay, Phys. Rev. C 78 (2008) 035504 [INSPIRE].

[31] A.S. Barabash, P. Hubert, A. Nachab, S.I. Konovalov and V. Umatov, Search for $\beta^{+} E C$ and ECEC processes in ${ }^{112}$ Sn, Phys. Rev. C 80 (2009) 035501 [arXiv:0909.1177] [INSPIRE].

[32] Z. Sujkowski and S. Wycech, Neutrinoless double electron capture: a tool to search for Majorana neutrinos, Phys. Rev. C 70 (2004) 052501 [hep-ph/0312040] [INSPIRE].

[33] F. Simkovic and M.I. Krivoruchenko, Mixing of neutral atoms and lepton number oscillations, Phys. Part. Nucl. Lett. 6 (2009) 298.

[34] J. Suhonen and M.T. Mustonen, Nuclear matrix elements for rare decays, Prog. Part. Nucl. Phys. 64 (2010) 235 [INSPIRE].

[35] M.I. Krivoruchenko, F. Simkovic, D. Frekers and A. Faessler, Resonance enhancement of neutrinoless double electron capture, Nucl. Phys. A 859 (2011) 140 [arXiv:1012.1204] [INSPIRE].

[36] D.-L. Fang et al., Evaluation of the resonance enhancement effect in neutrinoless double-electron capture in ${ }^{152} \mathrm{Gd},{ }^{164} \mathrm{Er}$ and ${ }^{180} \mathrm{~W}$ atoms, Phys. Rev. C 85 (2012) 035503 [arXiv: 1111.6862] [INSPIRE]. 
[37] T.R. Rodríguez and G. Martínez-Pinedo, Calculation of nuclear matrix elements in neutrinoless double electron capture, Phys. Rev. C 85 (2012) 044310 [arXiv:1203.0989] [INSPIRE].

[38] J. Suhonen, Nuclear matrix elements for the resonant neutrinoless double electron capture, Eur. Phys. J. A 48 (2012) 51 [inSPIRE].

[39] J. Kotila, J. Barea and F. Iachello, Neutrinoless double-electron capture, Phys. Rev. C 89 (2014) 064319 [arXiv:1509.01927] [INSPIRE].

[40] V. Weisskopf and E. Wigner, Over the natural line width in the radiation of the harmonius oscillator, Z. Phys. 65 (1930) 18 [INSPIRE].

[41] A. Galindo and P. Pascual, Quantum mechanics, Springer, Germany (1990).

[42] J. Bernabeu and M. Rosa-Clot, Dispersive approach to the nuclear Compton amplitude and exchange effects, Nuovo Cim. A 65 (1981) 87 [INSPIRE].

[43] M. Ericson and M. Rosa-Clot, Compton scattering and pion number in nuclei, Phys. Lett. B 188 (1987) 11 [INSPIRE].

[44] R.C. Hilborn, Einstein coefficients, cross sections, $f$ values, dipole moments, and all that, physics/0202029. .

[45] E. Schneidmiller and M. Yurkov, DESY note, private communication from M. Altarelli.

[46] K. Yamauchi et al., Nanofocusing of X-ray free-electron lasers by grazing-incidence reflective optics, J. Synchrotron Rad. 22 (2015) 592.

[47] H. Wallander and J. Wallentin, Simulated sample heating from a nanofocused X-ray beam, J. Synchrotron Rad. 24 (2017) 925.

[48] P. Roedig et al., High-speed fixed-target serial virus crystallography, Nat. Meth. 14 (2017) 805.

[49] E.A. Den Hartog and J.E. Lawler, Radiative lifetimes of neutral Samarium, J. Phys. B 46 (2013) 185001. 